\title{
Effect of Eudragit ${ }^{\circledR}$ RS 30D and Talc Powder on Verapamil Hydrochloride Release from Beads Coated with Drug Layered Matrices
}

\author{
Yasser El-Malah $^{1}$ and Sami Nazzal ${ }^{1,2}$
}

Received 2 July 2007; accepted 7 September 2007; published online 9 January 2008

\begin{abstract}
The aim of this study was to investigate the effect of Eudragit ${ }^{\circledR}$ RS 30D, talc, and verapamil hydrochloride on dissolution and mechanical properties of beads coated with "drug-layered matrices". This was accomplished with the aid of a three-factor multiple-level factorial design using percent drug release in 1 and $2 \mathrm{~h}, T_{50}$, tensile strength, brittleness, stiffness and toughness as the responses. Beads were coated in a fluidized-bed coating unit. Surface morphology and mechanical properties were evaluated by surface profilometry and texture analysis, respectively. No cracks, flaws and fissures were observed on the surfaces. The mechanical properties were dependent on the talc/polymer ratio. The release of verapamil from the beads was influenced by matrix components. Increasing the level of both talc and Eudragit decreased the percent drug released from $67 \%$ to $4.8 \%$ and from $80.7 \%$ to $6.7 \%$ in 1 and $2 \mathrm{~h}$, respectively, and increased $T_{50}$ from 0.8 to $25.7 \mathrm{~h}$. It was concluded that beads could be efficiently coated with "drug-layered matrices". The release of drug, however, depends on a balance between the levels of drug, talc, and polymer, whereby desired dissolution and mechanical properties could be controlled by the talc/polymer ratio and the level of drug loading.
\end{abstract}

KEY WORDS: drug layered matrix; Eudragit RS 30D; fluid bed coating; talc powder; verapamil hydrochloride.

\section{INTRODUCTION}

Multiparticulate dosage forms, such as beads or pellets, offer several advantages over conventional dosage forms. For example, their gastric emptying rate is more predictable and they are less likely to experience dose dumping $(1,2)$. When manufacturing a multiparticulate dosage forms, the drug is either incorporated within the beads through a process of extrusion and spheronization $(3,4)$ or simply adhered to the surface of the beads by a fluidized bed coating process $(5,6)$. When the drug is layered on the surface of the beads, it is subsequently coated with a control release membrane that would allow the beads attain the desired drug release profile. Alternatively, both the drug and the polymer required for controlled release could be coated on the beads simultaneously in a single step using a single coating dispersion (7). While less common, coating beads with a film forming material incorporating the drug, i.e., a layered drug matrix around the beads, is economic. It reduces the number of steps involved in the coating process meanwhile it provides flexibility in attaining the desirable drug release profile (8). Very few studies; however, were reported in the literature that address this alternative coating approach. A notable example is a study by

\footnotetext{
${ }^{1}$ Department of Basic Pharmaceutical Sciences, College of Pharmacy, University of Louisiana at Monroe, Monroe, Louisiana 71209-0497, USA.

${ }^{2}$ To whom correspondence should be addressed. (e-mail: nazzal@ ulm.edu)
}

Wu and McGinity (9) in which beads were coated with a blend of ibuprofen and Eudragit RS $30 \mathrm{D}$ as the film forming material. While ibuprofen was evaluated for its plasticizing effect, it was concluded that the release rate of ibuprofen was influenced by both the amount of ibuprofen in the polymeric film and the thickness of the coating layer. Another factor that is seldom considered for its effect on layered matrices is the presence of an anti-tacking agent. The addition of an antitacking agent such as talc to the film-forming material is often recommended to expedite processing by avoiding the agglomeration problems caused by polymeric particles during the coating process. The importance of talc, however, is more prominent in aqueous-based coating systems as the mechanism by which films are formed using these systems is more complex than those encountered with the organic-solvent based systems. In aqueous systems the coalescence of individual colloidal particles and the interdiffusion of polymeric particles must occur to form a continuous film (10). This is accomplished during the coating process as a result of water evaporation, which generates surface tension effects and capillary forces among polymer particles (11). The slow rate of water evaporation coupled with its high latent heat of vaporization, however, is also responsible for the tackiness problems experienced with these systems (12). Therefore, talc powder has been frequently used as an inert anti-tacking agent in aqueous-based acrylic colloidal dispersions when coating either single unit dosage forms (tablets) or multiparticulate units $(3,11,13)$. Maejima and McGinity (11) reported that high levels of talc powder up to $200 \%$, based on dry polymer weight, however, had a stabilizing effect on the release rate of 
Table I. Independent and Dependent Variables of the Full Factorial Design

\begin{tabular}{lclr}
\hline Independent Factors & Levels & & \\
\hline Coded values & -1 & 0 & 1 \\
$X_{1}$ : drug level $(\mathrm{g})$ & 1 & NA & 3 \\
$X_{2}:$ talc level $(\mathrm{g} \%)^{a}$ & 50 & 150 & 250 \\
$X_{3}$ : polymer level $(\mathrm{g})$ & 7.5 & NA & 13 \\
\hline
\end{tabular}

${ }^{a}$ Amount of talc based on dry polymer weight.

Dependent factors are $Y_{1}$ : percent drug released in $1 \mathrm{~h}, Y_{2}$ : percent drug released in $2 \mathrm{~h}, Y_{3}$ : tensile strength $\left(\mathrm{g} / \mathrm{mm}^{2}\right), Y_{4}$ : distance to fracture force (taken as an opposite indication to brittleness; $\mathrm{mm}$ ), $Y_{5}$ : stiffness $(\mathrm{g} / \mathrm{mm}), Y_{6}$ : toughness $(\mathrm{g} / \mathrm{mm}), T_{50}$ : time to $50 \%$ release $(\mathrm{h})$

theophylline from coated beads after storage at different conditions. This study implied that talc at higher levels might exhibit an effect other than its intended use as an anti-tacking agent. Talc was also shown to act as a thickness building agent in matrices $(8,14)$. Therefore, talc is expected to have a profound effect on the release rate of drugs from beads coated with drug layered matrices.

Based on the preceding discussion, the overall objective of this study was therefore to evaluate the dissolution and physical properties of $\mathrm{Nu}$-Pareil ${ }^{\circledR}$ beads directly coated with an aqueous dispersion consisting of a blend of verapamil HCL, Eudragit ${ }^{\circledR}$ RS 30 D, and talc. More specifically the goals of this study were to evaluate the effect of the level of verapamil HCL as a model drug, Eudragit ${ }^{\circledR}$ RS $30 \mathrm{D}$ as the film forming material, and talc powder on the mechanical strength of the beads and the dissolution rate of the drug. To accomplish this goal a three-factor, multiple-level full factorial design was used to evaluate the significance of each factor and their interaction effect on the responses.

\section{MATERIALS AND METHOD}

\section{Materials}

The aqueous dispersion of Eudragit ${ }^{\circledR}$ RS 30D [poly (ethyl acrylate, methyl methacrylate) trimethylammonioethyl methacrylate chloride] was obtained from RÖhm America Inc. (Piscataway, NJ). Triethyl citrate (TEC) was obtained from Morflex Chemical Co. (Greensboro, NC). Talc was purchased from Spectrum Quality Products (Gardena, CA). Verapamil HCL was supplied by BASF (Mount Olive, NJ). Nu-pareil sugar spheres NF mesh size 14/18 (1,000-1,410 $\mu$ m diameter) was provided by CHR Hansen (Mahwah, NJ). Nu-pareil Sugar Spheres NF are approximately spherical granules composed of sucrose and starch used as inert cores upon which the drug is coated. All chemicals and raw materials were used as received without further processing. Water used in this study was purified by Nanopure ${ }^{\circledR}$ Water System (Barnstead/Thermolyne, Dubuque, IA).

\section{Experimental Design}

In this study a three factor multiple level full factorial design was used to construct the response surface and the mathematical model for the tested variables. Full factorial design is commonly used to reveal the main effects and interaction effects between the independent variables of the experiment. For completely randomized factorial design with three factors at multiple levels, second order polynomial equation was generated using response surface methodology (RSM), which includes quadratic terms and two factor interaction that explain the non-linear nature of the response (15). A second order polynomial equation that describes the effect of independent factors on the response is expressed in the following form:

$$
\begin{aligned}
Y= & A_{0}+A_{1} X_{1}+A_{2} X_{2}+A_{3} X_{3}+A_{4} X_{1} X_{2}+A_{5} X_{1} X_{3} \\
& +A_{6} X_{2} X_{3}+A_{7} X_{1}^{2}+A_{8} X_{2}^{2}+A_{9} X_{3}^{2}+E
\end{aligned}
$$

Where $Y$ is a response, $X_{1}-X_{3}$ are the independent factors, $A_{\mathrm{o}}$ is a constant, $A_{1}-A_{9}$ are the coefficients of the respective variables and their interaction terms, and $E$ is an error term. Results of statistical analysis are usually considered significant if their corresponding $P$ values are less than 0.05. In this study the levels of the independent factors that were used to construct a full factorial design is given in Table I. A total of 12 experimental runs were prepared and tested according to the full factorial design.

\section{Preparation of Beads Coated with Drug Layered Matrices}

A total of 12 coating dispersions were prepared according to the amounts specified by the experimental design. The

\begin{tabular}{|c|c|c|c|c|c|c|c|c|c|c|c|c|c|}
\hline Run & Drug Level & Talc Level & Polymer Level & $Y_{1}(\%)$ & $Y_{2}(\%)$ & $T_{50}(\mathrm{~h})$ & $Y_{3}$ & $Y_{4}$ & $Y_{5}$ & $Y_{6}$ & $R_{\mathrm{q}}(\mathrm{nm})$ & $R_{\mathrm{a}}(\mathrm{nm})$ & $\begin{array}{l}\text { Bead Diameter } \\
(\mathrm{mm})\end{array}$ \\
\hline 1 & 1 & 0 & -1 & 83.3 & 86.1 & 0.40 & 1,599 & 0.299 & 9,759 & 496.7 & 353 & 293 & 1.53 \\
\hline 2 & 1 & 1 & -1 & 78.6 & 82.0 & 0.40 & 1,443 & 0.284 & 9,077 & 398.8 & 505 & 381 & 1.51 \\
\hline 3 & -1 & 1 & -1 & 8.50 & 12.8 & 12.4 & 1,059 & 0.281 & 8,019 & 357.6 & 788 & 621 & 1.64 \\
\hline 4 & -1 & 0 & 1 & 8.96 & 12.2 & 16.2 & 1,076 & 0.394 & 8,959 & 782.4 & 1,155 & 894 & 2.04 \\
\hline 5 & 1 & 0 & 1 & 40.3 & 72.4 & 1.42 & 1,261 & 0.402 & 7,699 & 664.1 & 387 & 316 & 1.77 \\
\hline 6 & 1 & -1 & -1 & 98.0 & 99.0 & 0.25 & 799.6 & 0.158 & 8,720 & 127.3 & 517 & 419 & 1.48 \\
\hline 7 & -1 & 0 & -1 & 14.5 & 36.3 & 2.88 & 1,511 & 0.257 & 9,991 & 349.0 & 357 & 280 & 1.47 \\
\hline 8 & 1 & 1 & 1 & 37.1 & 68.5 & 1.42 & 1,125 & 0.367 & 9,428 & 706.1 & 698 & 569 & 1.97 \\
\hline 9 & -1 & 1 & 1 & 4.80 & 6.72 & 25.7 & 1,408 & 0.342 & 8,668 & 562.5 & 913 & 723 & 1.65 \\
\hline 10 & -1 & -1 & 1 & 16.3 & 29.2 & 3.02 & 1,409 & 0.279 & 8,978 & 333.7 & 440 & 346 & 1.49 \\
\hline 11 & 1 & -1 & 1 & 90.9 & 98.2 & 0.25 & 1,468 & 0.316 & 8,639 & 416.4 & 568 & 415 & 1.54 \\
\hline 12 & -1 & -1 & -1 & 67.0 & 80.7 & 0.83 & 1,080 & 0.178 & 9,735 & 146.7 & 744 & 555 & 1.43 \\
\hline
\end{tabular}

Table II. The Randomized Runs of the Full Factorial Design and the Observed Responses 
composition of the coating dispersions is given in Table II. Briefly, talc powder was first homogenized with a blend of water and $20 \%$ triethyl citrate, based on the dry polymer weight, for $10 \mathrm{~min}$. Then it was added to the Eudragit ${ }^{\circledR}$ RS 30D aqueous dispersions under agitation. Verapamil HCL was dissolved in purified water and then added to the coating dispersion. Masterflex ${ }^{\circledR}$ Digi-Static ${ }^{\circledR}$ pump (Cole-Parmer Instrument Company, Veronon Hill, Illinois) was used to feed the formulations to the fluidized-bed coater (MFL.01, Vector Corporation, Marion, IA). To ensure that only dry air is flowing into the system, a Hankison air trap and several in-line filters were placed between the fluid-bed and the compressor. Loading charge of $20 \mathrm{~g} \mathrm{Nu}$-pareil ${ }^{\circledR}$ sugar spheres, of mesh size 14/18 $(1,400-1,000 \mu \mathrm{m}$ in diameter), was used in this study. Processing parameters (Table III) were kept constant for all runs. The coating suspension was agitated during the coating process to maintain homogeneity in the formulation. At the end of the coating process, coated beads were cured in an oven at $40^{\circ} \mathrm{C}$ for $24 \mathrm{~h}$.

\section{Morphology of Beads Coated with Drug Layered Matrices}

The surface view of the intact and divided beads was recorded using a VHX-600 Digital Microscope with an optical diffused lighting source (KEYENCE Corp., Woodcliff Lake, NJ). Surface roughness of the beads coated with a "film matrix" was measured using Alpha-Step 1Q stylus contact surface profiler (model AS-1Q, KLA-Tencor Corporation, San Jose, CA). The instrument was adapted with a diamond stylus which was programmed to scan a length of $750 \mu \mathrm{m}$ of the pellet surface at a scanning speed $20 \mu \mathrm{m} / \mathrm{s}$. In each experiment, the $R_{\mathrm{q}}(\mathrm{RMS})$ and $R_{\mathrm{a}}$ (Average) surface parameters were determined.

\section{Content Uniformity}

To extract verapamil HCL, an accurately weighed sample of the beads ( $1.5 \mathrm{~g}$ ), from each run, was ground and transferred to a $500 \mathrm{ml}$ volumetric flask containing purified water. The flask was sonicated for $30 \mathrm{~min}$. After $24 \mathrm{~h}$ of storage at room temperature, the aqueous dispersion was then filtered, diluted, and analyzed spectrophotometrically at $275 \mathrm{~nm}$ (Cary 50 probe UV spectrophotometer, Varian Inc, Cary, NC). All assays were carried out in triplicate and the mean value was reported.

Table III. Coating Process Parameters

\begin{tabular}{ll}
\hline \multicolumn{2}{c}{ Parameters } \\
\hline Nozzle diameter & $0.029 \mathrm{~mm}$ \\
Wurster insert & Bottom spray \\
Atomization air pressure & $25 \mathrm{psi}$ \\
Preheating temperature & $32^{\circ} \mathrm{C}$ \\
Preheating time & $5 \mathrm{~min}$ \\
Batch size & $20 \mathrm{~g}$ \\
Spray rate & $0.6 \mathrm{ml} / \mathrm{min}$ \\
Inlet temperature & $38^{\circ} \mathrm{C}$ \\
Bed temperature & $29-30^{\circ} \mathrm{C}$ \\
Inlet air & $220 \mathrm{LPM}$ \\
\hline
\end{tabular}

\section{Dissolution Study}

Dissolution studies on a fixed weight of $1.5 \mathrm{~g}$ of the beads were performed in triplicates using a USP type II (paddle) dissolution apparatus (VK 7000, Varian Inc., Cary, NC). The dissolution medium consisted of $900 \mathrm{ml}$ of purified water, which was maintained at $37^{\circ} \mathrm{C}$ and agitated at $75 \mathrm{rpm}$. Samples $(5 \mathrm{ml})$ were withdrawn at predetermined time intervals, filtered and analyzed spectrophotometrically at $275 \mathrm{~nm}$. From the result, the cumulative percent drug release-time profiles were determined. Dissolution experiments were performed in triplicates, unless otherwise stated.

\section{Mechanical Properties of an Individual Pellet}

The mechanical properties of a single pellet were determined in terms of tensile strength, brittleness, Young's modulus and toughness using a TA.XTPlus texture analyzer (Texture Technologies Corp., Scarsdale, NY/Stable Micro Systems, Godalming, Surrey, UK) fitted with a $1 \mathrm{~mm}^{2}$ flat stainless steel probe. Beads were compressed at a rate of $0.10 \mathrm{~mm} / \mathrm{s}$ whereby mechanical properties were estimated from the generated force-displacement profiles.

\section{RESULTS AND DISCUSSION}

\section{Statistical Design and Analysis}

Full factorial multiple levels experimental design was used in this study to evaluate the main effects and the interaction terms of the test independent factors. The levels of each independent factor evaluated and the investigated responses are listed in Table I. Verapamil and polymer effect were evaluated at two levels only whereas talc was evaluate at three levels. This was based on preliminary studies (unpublished data), which showed that polymer and verapamil exert linear effect on the responses. A 12-run factorial design was generated using STATGRAPHICS plus 5 statistical experiment design software (Manugistics Incorporation, Rockville, MA). The 12 runs in the experimental design along with the observed responses are shown in Table II. A standard significance level of $\alpha=0.05$ was selected in this study. This indicates that the effect of the test variable will be considered significant if its $P$ value is less than 0.05 . Table IV shows the model parameters obtained from analysis of variance (ANOVA) for the responses $Y_{1}-Y_{6}$ and $T_{50}$. Table IV was used to construct the models that describe the effect of the test variables on the responses $\left(Y_{1}-Y_{6}\right.$ and $\left.T_{50}\right)$. Regression equations for the fitted models are presented in Table V. The magnitude and direction of the factor coefficient in the equations explains the nature of effect of factors $\left(X_{1}-X_{3}\right)$ on the response. Factors with coefficients of greater magnitude show high effect on the response. The sign of the coefficient indicates the direction by which the factor influences the response. The effect of factors on the responses is further elucidated in subsequent sections.

\section{Morphology of Beads Coated with Drug Layered Matrices}

To investigate the effect of $X_{1}$ (amount of verapamil HCL), $X_{2}$ (amount of talc powder) and $X_{3}$ (amount of Eudragit RS 30D) on the surface topography and roughness 
Table V. Regression Equations of the Fitted Models

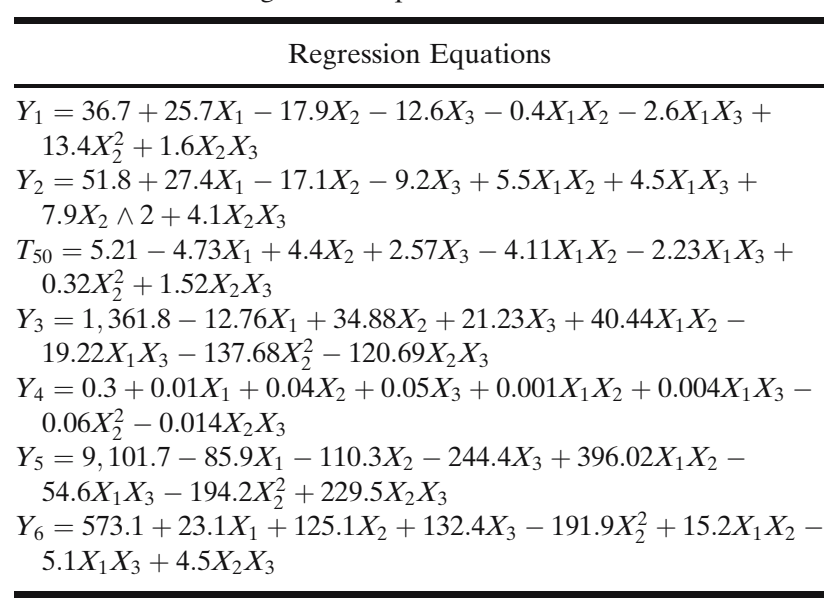

of the beads, microscopic imaging and contact profilometry were carried out on intact and scalpel-divided beads. A representative microscopic image is given in Fig. 1. It was observed that an increase in the level of study factors resulted in an increase in the thickness of the matrix layer around the core. At any factor-level combination, the layer around the core homogenously surrounded the core indicating the efficiency of the coating process. No cracks or fissures were observed at any combination levels of the study factors. When the amount of verapamil HCL was increased, at a constant level of Eudragit ${ }^{\circledR}$ RS 30D and talc, microscopic white particles of verapamil HCL were observed on the surface of the beads. These particles would serve as entrance or exit points for the dissolution medium to the deeper region of the matrix after the release of the drug from the surface of the beads.

The effect of the study factors on the surface roughness of the beads coated with the film matrix was determined by measuring the surface parameters $R_{\mathrm{q}}$ (RMS) and $R_{\mathrm{a}}$ (Average). $R_{\mathrm{q}}$ (RMS) is the Root-Mean-Square or geometric average deviation of the profile from the mean line measured in the sampling length. $R_{\mathrm{a}}$ (Average) is the arithmetic average deviation of the absolute values of the roughness profile from the mean line or center line. Measured $R_{\mathrm{q}}$ and $R_{\mathrm{a}}$ values for all beads were in the nano-meter range (Table II), which indicates smoothness of the surfaces and absence of cracks. Figure 2 shows the effect of increasing the amount of $X_{2}$ and $X_{3}$ on $R_{\mathrm{q}}$ and $R_{\mathrm{a}}$ roughness parameters. Both $R_{\mathrm{q}}$ and $R_{\mathrm{a}}$ increased with an increase in the levels of $X_{2}$ and $X_{3}$ which

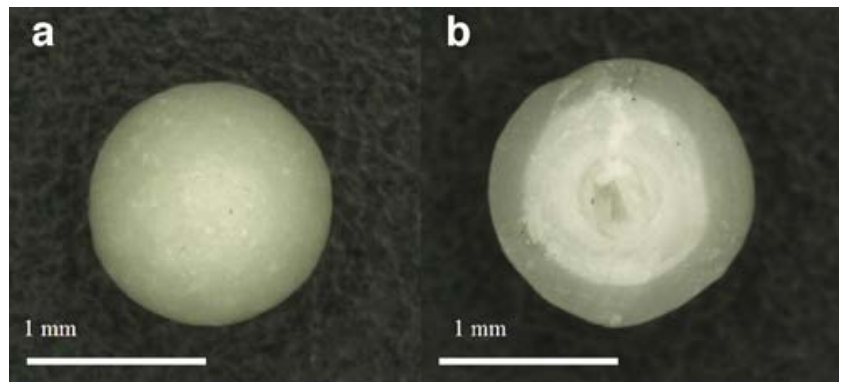

Fig. 1. Microscopic image of the a external surface of intact pellet and $\mathbf{b}$ internal surface of divided pellet 
a

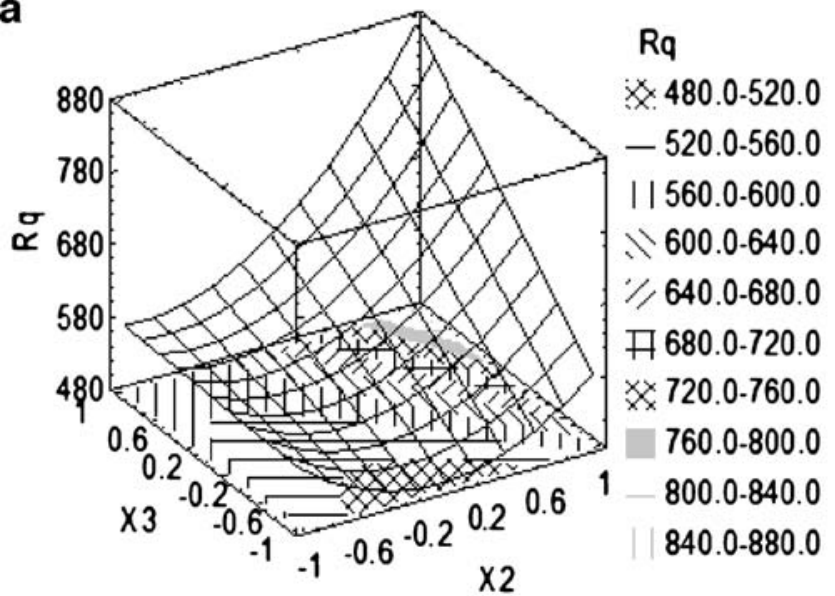

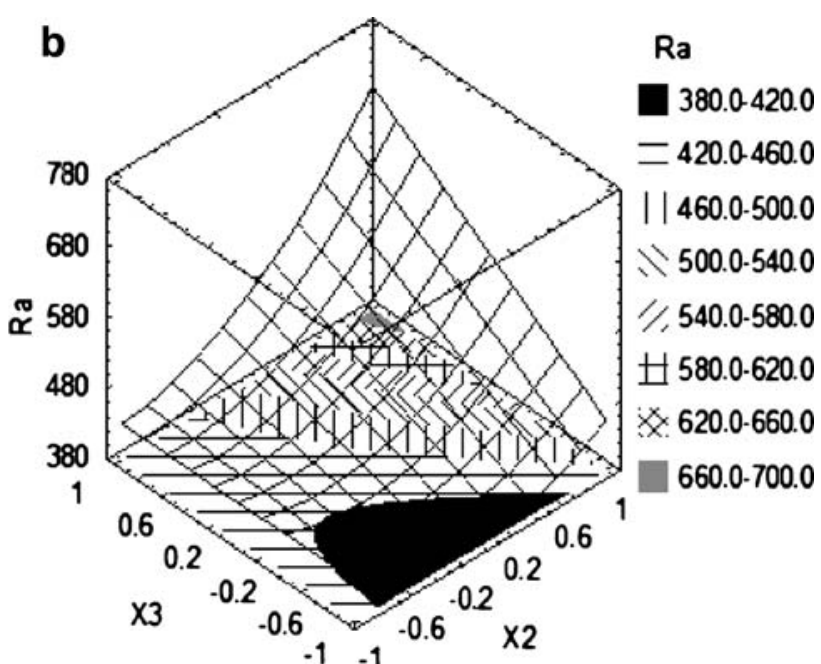

Fig. 2. Response surface plots showing the effect of talc level $\left(X_{2}\right)$ and polymer level $\left(X_{3}\right)$ on a $R_{\mathrm{q}}$ roughness parameter and $\mathbf{b} R_{\mathrm{a}}$ roughness parameter

indicates that an increase in the amount of talc powder and Eudragit ${ }^{\circledR}$ RS 30D increases the surface roughness of the beads coated with film matrices.

\section{Effect of Study Factors on Verapamil HCL Release}

The influence of the level of Eudragit ${ }^{\circledR}$ RS 30D, talc and verapamil HCL on the release properties of verapamil HCL from the beads coated with drug layered matrices was determined from their dissolution profiles, which are given in Fig. 3. The effect of each factor on the responses is discussed in the following sub-sections. Table IV shows the model parameters obtained from analysis of variance (ANOVA) for the responses. The models as fitted explain $92.15 \%, 96.72 \%$, and $96.75 \%$ of the variability in $Y_{1}, Y_{2}$, and $T_{50}$, respectively, which were the primary responses considered in this study.

\section{Effect of Eudragit ${ }^{\circledR}$ RS 30D}

Eudragit ${ }^{\circledR}$ RS 30D is a methacrylic ester copolymer available as a latex-like aqueous dispersion. The commercial aqueous dispersion, with $30 \%$ polymer content is referred to as 30 D (16). Due to its ability to form a continuous film upon spraying and drying, Eudragit ${ }^{\circledR}$ RS 30 D has been frequently used as a release controlling film (3,16-19). In the present study Eudragit ${ }^{\circledR}$ RS 30 D was used as a film and matrix forming material. Statistical analysis was carried out to evaluate the effect of Eudragit ${ }^{\circledR}$ RS 30D on the dissolution responses $Y_{1}, Y_{2}$ and $T_{50}$. Analysis of variance showed that Eudragit ${ }^{\circledR}$ RS 30D had an insignificant effect on $Y_{1}$ but a negative and significant effect on $Y_{2}$ and a positive and significant effect on $T_{50}$. At low levels of talc and verapamil HCL, increasing the amount of Eudragit ${ }^{\circledR}$ RS 30D from 7.5 to $13 \mathrm{~g}$ decreased the percent cumulative amount of drug released in $1 \mathrm{~h}\left[Y_{1}\right]$ from $67.02 \%$ to $16.29 \%$. However, at high level of verapamil HCL, an increase in the amount of Eudragit ${ }^{\circledR}$ RS 30D only slightly decreased $Y_{1}$ from $98.01 \%$ to $90.89 \%$. These findings indicated that the effect of Eudragit ${ }^{\circledR}$ RS 30D is only predominant at low levels of verapamil HCL. This could be explained by the fact that verapamil HCL is a water soluble drug. Upon dissolution, the drug on the surface of the beads dissolves and leaches out creating a porous
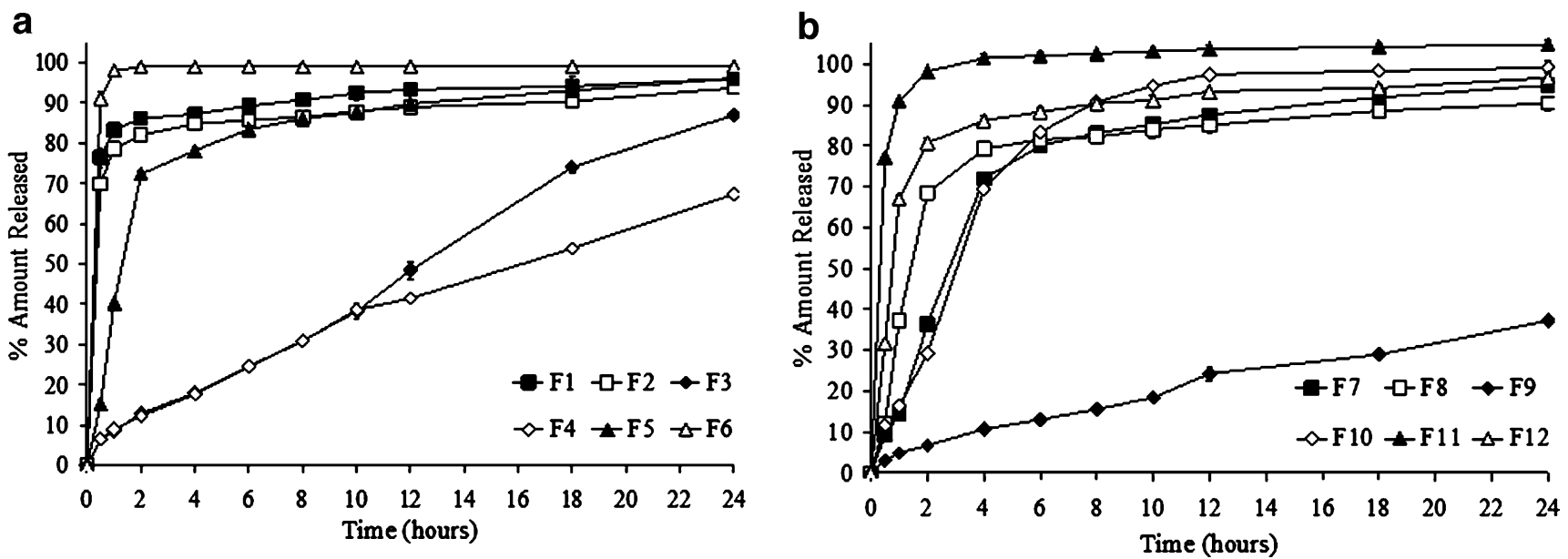

Fig. 3. Dissolution profiles of verapamil $\mathrm{HCl}$ from pellets coated with drug-layered matrices: a runs $1-6$ and b runs $7-12$ 

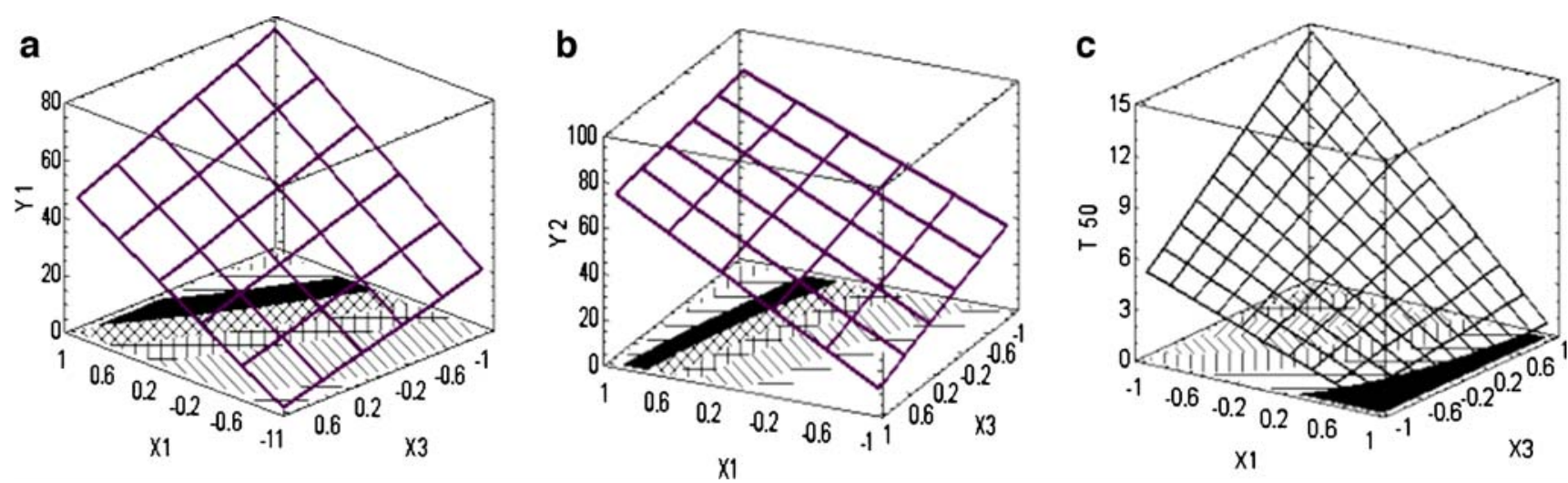

Fig. 4. Response surface plots showing the effect of verapamil $\mathrm{HCl}$ level $\left(X_{1}\right)$ and polymer level $\left(X_{3}\right)$ on responses a $Y_{1}$, b $Y_{2}$, and $\mathbf{c} T_{50}$

matrix through which remaining drug readily diffuses into the dissolution medium. Increasing the level of Eudragit ${ }^{\circledR}$ polymer, however, forms a rigid matrix, which decreases the mobility of drug molecules. This in turn decreases the number of pores through which the drug could diffuse into the dissolution medium. This decrease in porosity was observed in the dissolution profiles by the decrease in the cumulative percent verapamil released. The effect of Eudragit ${ }^{\circledR}$ RS 30D was insignificant in the first hour, during which the drug is mainly released from the surface of the beads. In the second hour $\left(Y_{2}\right)$, however, drug primarily diffused from the interior of the matrix, which was affected by the decreasing number of pores due to the increase in Eudragit ${ }^{\circledR}$ RS 30D amount. Therefore increase in the level of Eudragit ${ }^{\circledR}$ RS 30D had a negative and significant effect on $Y_{2}$. The overall effect of Eudragit ${ }^{\circledR}$ RS 30D level on dissolution process is further observed by the increase in time required to release $50 \%$ of verapamil HCL $\left(T_{50}\right)$.

\section{Effect of Verapamil HCL}

The level of verapamil HCL in the drug layered matrix was found to have a significant effect on the responses $Y_{1}$ [percent amount released in $1 \mathrm{~h}$ ], $Y_{2}$ [percent amount released in $2 \mathrm{~h}$ ] of verapamil HCL and $T_{50}$ [time required to $50 \%$ verapamil HCL release]. The effect of verapamil HCL $\left[X_{1}\right]$ and Eudragit ${ }^{\circledR}$ RS $\left[X_{3}\right]$, at mid level of talc $\left[X_{2}\right]$, on $Y_{1}, Y_{2}$ and $T_{50}$ are shown in Fig. 4. At low polymer level $\left[X_{3}\right]$, increasing verapamil HCL $\left[X_{1}\right]$ from 1 to $3 \mathrm{~g}$ increased $Y_{1}$ from $14.5 \%$ to
$83 \%$ and $Y_{2}$ from $36 \%$ to $86 \%$ while; $T_{50}$ was decreased from about $5 \mathrm{~h}$ to less than half hour. At high levels of $X_{3}$, as the level of $X_{1}$ increased from 1 to $3 \mathrm{~g}, Y_{1}$ increased from $9 \%$ to $40 \%$ and $Y_{2}$ increased from $12.2 \%$ to $72.3 \%$ while $T_{50}$ decreased from $14 \mathrm{~h}$ to only $1 \mathrm{~h}$. Increasing the amount of verapamil HCL, at fixed levels of $X_{2}$ and $X_{3}$, also decreased the average time required to release at least $90 \%$ of the drug from $8 \mathrm{~h}$ to less than $2 \mathrm{~h}$. The significant effect of verapamil HCL on $Y_{1}, Y_{2}$, and $T_{50}$ demonstrates that soluble drugs, such as verapamil HCL (solubility $1 \mathrm{~g} / 16 \mathrm{ml}$ ), act as pore formers in film matrices. When coated beads are exposed to the dissolution medium, the drug dissolves and leaches out of the matrix, which creates pores in the matrix through which more drug continue to diffuse into the dissolution medium. This effect, however, is more pronounced at high drug levels, which explains the increase in the cumulative amount released with time.

\section{Effect of Talc Powder}

Traditionally talc powder is used as an anti-tacking agent at concentrations ranging from $25 \%$ to $100 \%$, based on the weight of dry polymer, to facilitate the coating process (11). Talc has the ability to decrease the agglomeration tendency of pellets when coated with aqueous polymeric dispersions. In addition to its effect as an anti-adherent, talc was used as a matrixforming material in this study. Talc powder had a significant negative effect on both $Y_{1}$ and $Y_{2}$, and a significant positive effect on $T_{50}$. The effect of talc powder $\left[X_{2}\right]$ and Eudragit ${ }^{\circledR}$ RS
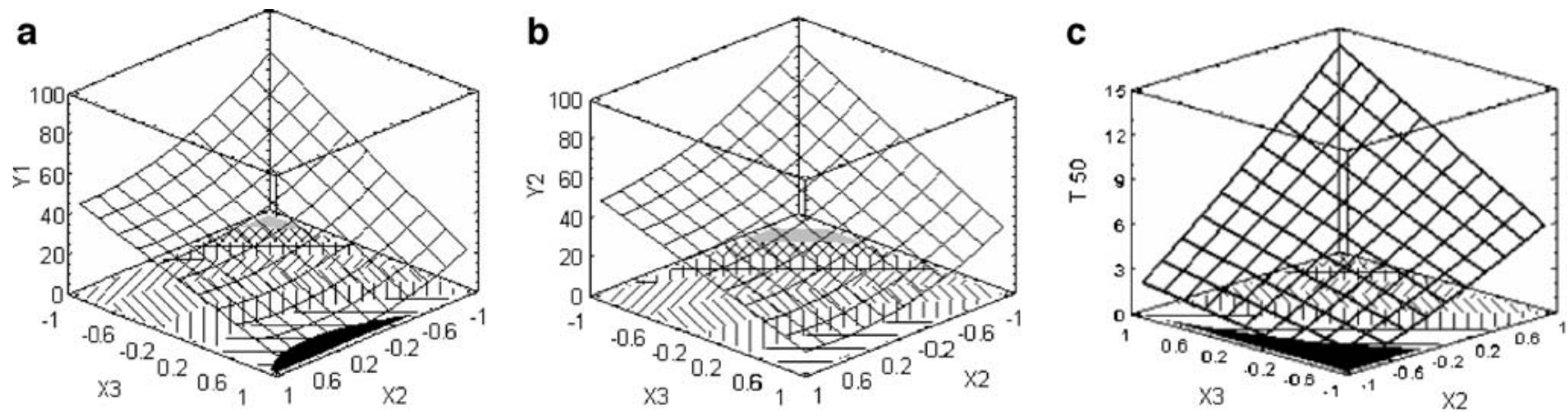

Fig. 5. Response surface plots showing the effect of talc level $\left(X_{2}\right)$ and polymer level $\left(X_{3}\right)$ on responses a $Y_{1}$, b $Y_{2}$, and $\mathbf{c} T_{50}$ 


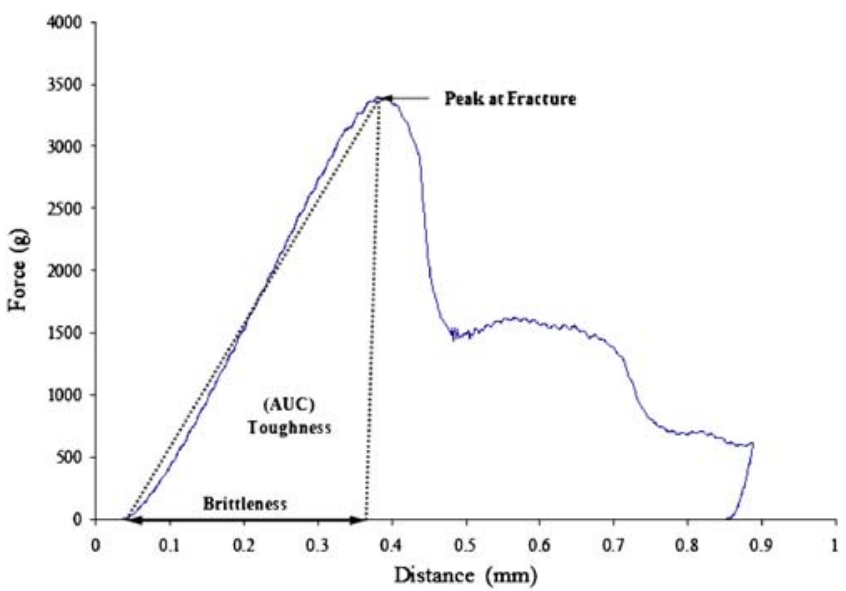

Fig. 6. A representative force-displacement profile obtained from the texture analyzer
[X $\left.X_{3}\right]$ on $Y_{1}, Y_{2}$, and $T_{50}$ is shown in Fig. 5. At mid level of verapamil HCL $\left[X_{1}\right]$ and low level of $X_{3}$, increasing $X_{2}$ from $50 \%$ to $250 \%$, based on dry polymer weight, decreased $Y_{1}$ from $80 \%$ to $40 \%$ and $Y_{2}$ from $87 \%$ to $47 \%$ while $T_{50}$ was increased from less than 1 to $6 \mathrm{~h}$. Increasing talc content from $50 \%$ to $250 \%$ also extended the time required to release at least $90 \%$ of verapamil HCL from 8 to $24 \mathrm{~h}$. This finding illustrates the ability of talc powder to modulate the release profile of the drug. The coefficient of talc effect on both responses $Y_{1}$ and $Y_{2}$ was approximately the same which indicates that talc influenced both responses by a similar mechanism. At high levels of talc, the matrix would consist primarily of talc and polymer latex. As the particle size of the polymer latex is much smaller than that of talc, consequently talc at higher levels would form the skeleton of the drug-layered matrix $(12,20)$, thereby creating a diffusing barrier to verapamil HCL.
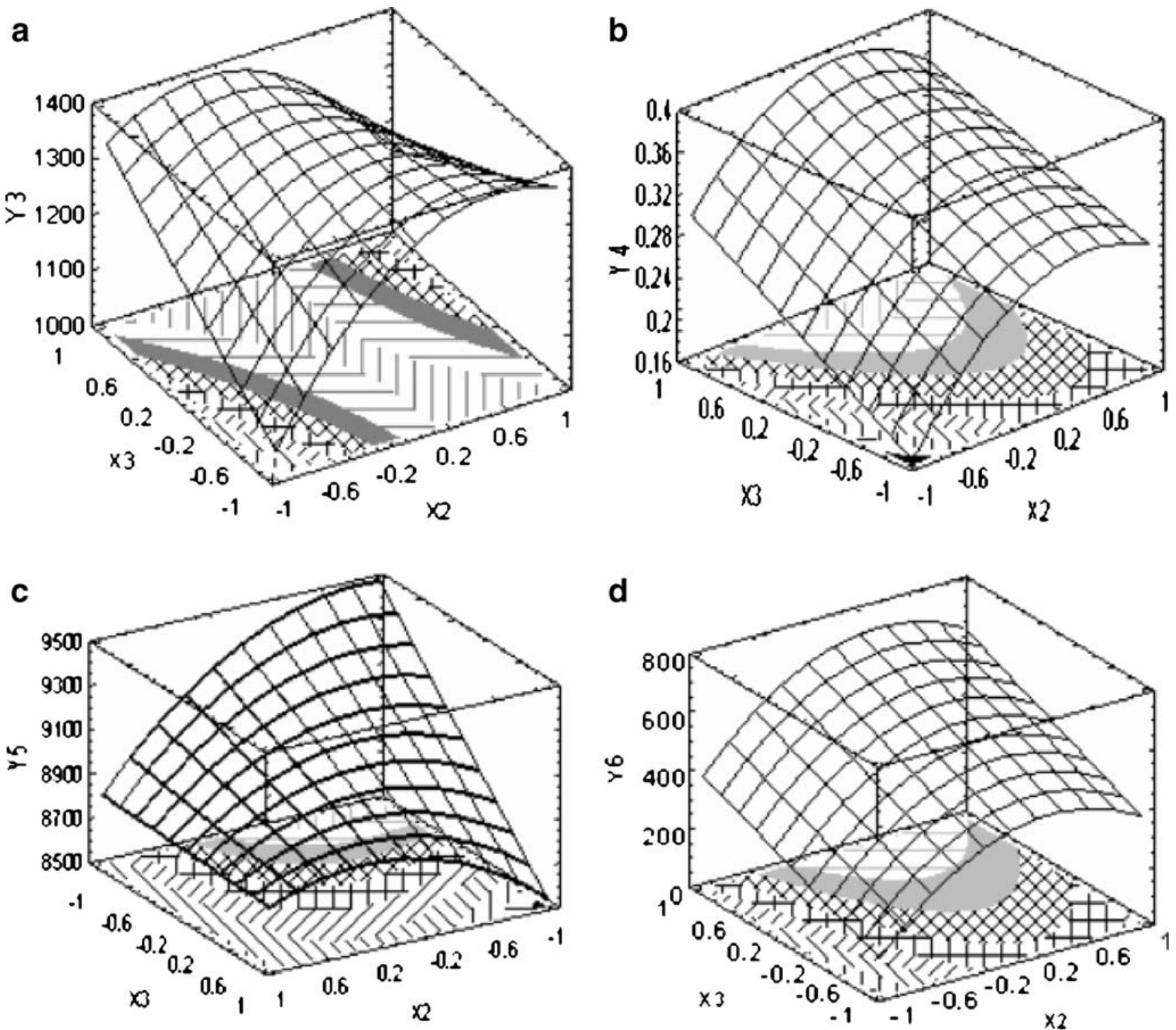

Fig. 7. Response surface plots showing the effect of talc level $\left(X_{2}\right)$ and polymer level $\left(X_{3}\right)$ on a tensile strength $\left(Y_{3}\right)$, b distance to fracture force $\left(Y_{4}\right)$, c stiffness $\left(Y_{5}\right)$, and $\mathbf{d}$ toughness $\left(Y_{6}\right)$ 


\section{Effect of Study Factors on the Mechanical Properties of the Beads}

Beads coated with a drug matrix must be resistant to physical stresses exerted by both external force, such as those experienced during shipping and packaging, and internal pressure due to osmosis and peristaltic movement within the gastro-intestinal tract. Such forces could rupture the matrix and result in dose dumping. The mechanical properties of the coated beads could be therefore used as an indirect measure of their ability to withstand such pressures. When investigating the mechanical properties of a single bead, it was assumed that the bead is spherical to avoid scatter in the data and have no crack, flaws or fissures. Also the bead was assumed to be isotropic, which means the properties of the pellet do not change by changing its direction (21). Texture analyzer was utilized to investigate the mechanical properties of a single pellet. All beads coated with verapamil HCL matrix showed plastic deformation. A representative force-distance curve obtained from the instrument is depicted in Fig. 6. From the plot four parameters were determined; fracture force, distance to fracture force, stiffness, and toughness. The two main parameters that were investigated in greater detail were the maximum force at fracture and the distance to fracture force. Distance to fracture force was taken as an indication of brittleness. The less the distance to fracture forces, the more the brittleness of the bead. Tensile strength of a single bead was calculated using Kuno's equation (22):

$$
\mathrm{TS}=4 P / \pi D^{2}
$$

Where $P$ is the maximum force at fracture and $D$ is the diameter of the pellet. Stiffness was obtained from the slope of the regression line to fracture force. Stiffness is defined as the ability of the bead to resist deformation. The fourth parameter which was estimated in this study was toughness, which is defined as the total work required for the pellet to undergo deformation (21). Toughness was measured from the area under force-distance curve from zero to maximum force as illustrated in Fig. 6.

\section{Effect of Talc and Eudragit ${ }^{\circledR} \mathrm{RS}$ on $Y_{3}$ and $Y_{4}$}

Figure 7 ( $\mathrm{a}$ and $\mathrm{b}$ ) show the effect of talc powder $\left[X_{2}\right]$, and Eudragit ${ }^{\circledR}$ RS $30 \mathrm{D}\left[X_{3}\right]$, on the responses, $Y_{3}$ (tensile strength) and $Y_{4}$ (distance to fracture force). None of study factors had significant effect on $Y_{3}$, whereas both talc $\left[X_{2}\right]$ and Eudragit ${ }^{\circledR}$ RS $\left[X_{3}\right]$ had a significant positive effect on $Y_{4}$, therefore they had a negative significant effect on brittleness. The least tensile strength values were observed at low levels of $X_{2}$ and $X_{3}$. The effect of increasing $X_{3}$ on tensile strength depends on the level of $X_{2}$. It increases tensile strength at low and mid levels of $X_{2}$, while decreases tensile strength at high level of $X_{2}$. On the other hand, at any level of $X_{2}$ increase level of $X_{3}$ led to a linear increase in the distance to fracture force therefore, decrease brittleness. Talc however had a non-linear effect on $Y_{3}$ and $Y_{4}$. Initially $Y_{3}$ and $Y_{4}$ increased with an increase in talc content and then gradually declined with further increase in talc content. This finding could be explained as follow. At low level of talc powder, the matrix would consist mainly of bonded polymer particles. Plasticization of Eudragit ${ }^{\circledR}$ RS 30D with $20 \%$ triethyl citrate; based on dry polymer weight, however, results in soft thin matrix, which results in low tensile strength and high brittleness. As the talc level increases, thickness of the matrix increases, and the bonds between the polymer and talc increases, this leads to an increase in tensile strength and decreases brittleness. Further increase in the talc level, however, results in a thick matrix, which is formed mainly of talc with dispersed polymer particles sufficient to bind the matrix. This decreases the tensile strength and distance to force at fracture i.e. increase brittleness of the beads. These findings illustrate that both tensile strength and brittleness are dependent on thickness and the balance between Eudragit ${ }^{\circledR}$ RS and talc in the matrix.

\section{Effect of Talc and Eudragit ${ }^{\circledR}$ RS on $Y_{5}$ and $Y_{6}$}

Figure 7 (c and d) illustrate the effect of talc powder $\left[X_{2}\right]$ and Eudragit ${ }^{\circledR}$ RS 30D $\left[X_{3}\right]$ on the responses $Y_{5}$ (stiffness) and $Y_{6}$ (toughness) of the pellets coated with the drug layered matrix. Within the limits of this study, none of the factors had a significant effect on $Y_{5}$. Maximum stiffness; however, was observed at low levels of $X_{2}$ and $X_{3}$. Increasing $X_{2}$ or $X_{3}$ led to a decrease in $Y_{5} . X_{2}$ and $X_{3}$ however had a significant positive effect on pellet toughness $\left[Y_{6}\right]$. Lowest toughness value was observed when beads were coated with a matrix at low levels of $X_{2}$ and $X_{3}$. As $X_{2}$ and $X_{3}$ increased the toughness increased. These findings could be explained on the basis of the balance of bonds formed between the matrix components as mentioned above.

\section{CONCLUSION}

Controlled drug release could be achieved at lower drug loading by manipulating the levels of talc and polymer. The effect of talc/polymer ratio on the dissolution process was evident by its impact on $T_{50}$. Mechanical properties of the beads were also influenced by the talc/polymer ratio. Within the limits evaluated in this study, increasing talc and or polymer resulted in improved mechanical properties, which further supports the idea that the dissolution and mechanical properties of beads coated with a drug-layered matrix could be readily manipulated by balancing the matrix-forming ingredients.

\section{REFERENCES}

1. T. Osawa, T. Takahata, H. Takahata, M. Maejima, M. Kbayashi, T. Noda. Sigmoidal releasing system (SRS) as a novel oral controlled release formulation. Proceedings of the Oral Controlled Release Formulation, 1991, pp 405-406.

2. N. Follonier and E. Doelker. Biopharmaceutical comparison of oral multiple-unit and single unit sustained release dosage forms. S.T.P. Pharma Sci 2:141-158 (1992).

3. C. Wu and J. W. McGinity. Influence of an enteric polymer on drug release rates of theophylline from pellets coated with Eudragit RS 30 D. Pharm. Dev. Technol 8:103-110 (2003).

4. W. Zheng and J. W. McGinity. Influence of Eudragit NE 30 D blended with Eudragit L 30 D-55 on the release of phenylpropanolamine hydrochloride from coated pellets. Drug Dev. Ind. Pharm 29:357-366 (2003).

5. S. Vaithiyalingam, I. K. Reddy, and M. A. Khan. Aquoeousbased cellulose acetate butyrate dispersion: Screening of process 
and formulation variables to obtain verapamil HCL-controlled release beads. Part. Sci. Tech 19:131-144 (2001).

6. A. Akhgari, H. A. Garekani, F. Sadeghi, and M. Azimaie. Statistical optimization of indomethacin pellets coated with $\mathrm{pH}$ dependent methacrylic polymers for possible colonic drug delivery. Int. J. Pharm 305:22-30 (2005).

7. N. Ur-rahman and K. H. Yuen. Eudragit NE 40-drug mixed coating system for controlling drug release of core pellets. Drug Dev. Ind. Pharm 31:339-347 (2005).

8. Y. El-Malah and S. Nazzal. Fluid bed coating: The utility of dual programmable pumps for controlled gradient drug deposition on pellets. Int. J. Pharm 337:361-364 (2007).

9. C. Wu, and J. W. McGinity. Influence of ibuprofen as a solid-state plasticizer in Eudragit ${ }^{\circledR}$ RS 30 D on the physicochemical properties of coated beads. AAPS PharmSciTech. 2(4), article 24 (2001).

10. Y. Fukumori. Coating of multiparticulates using polymeric dispersion. In I. Ghebre-Sellassie (ed.), Multiparticulate Oral Drug Delivery-Drugs and Pharmaceutical Science, Vol. 65, Marcel Dekker, New York, NY, 1994, pp. 79-117.

11. T. Maejima and J. W. McGinity. Influence of film additives on stabilizing drug release rates from pellets coated with acrylic polymers. Pharm. Dev. Technol 6:211-221 (2001)

12. L. S. C. Wan and W. F. Lai. The influence of antitack additives on drug release from film-coated granules. Int. J. Pharm 94:39-47 (1993).

13. E. Hamed and A. Sakr. Effect of curing condition and plasticizer level on the release of highly lipophilic drug from coated multiparticulate drug delivery system. Pharm. Dev. Technol 8:397-407 (2003).

14. D. C. Scott and R. G. Hollenbeck. Design and manufacture of a zero-order sustained release pellet dosage form through nonuni- form drug distribution in a diffusional matrix. Pharm. Res 8:156161 (1991).

15. R. MyersD. Montgomery. Response surface methodology: process and product optimization using designed experiments1Wiley Interscience, New York, NY, 1995.

16. K. O. R. Lehmann. Chemistry and application properties of polymethacrylate coating systems. In J. W. McGinity (ed.), Aqueous Polymeric Coating for Pharmaceutical Dosage Forms, Second edition (revised and expanded), Vol 79, Marcel Dekker, New York, NY, 1997, pp. 112-144.

17. R. G. Bodmeier, R. E. X. Sarabia, and P. F. Skultety. The influence of buffer species and strength on diltiazim-HCL release from beads coated with the aqueous cationic polymer dispersion, Eudragit RS, RL 30D. Pharm. Res 3:52-60 (1996).

18. B. C. Lippold and R. Monells Pages. Control and stability of drug release from diffusion pellets coated with the aqueous quaternary polymethacrylate dispersion Eudragit RS 30 D. Pharmazie 56:477-483 (2001).

19. H. S. Alkhatib and A. Sakr. Optimization of methacrylic acid ester copolymer blends as controlled release coating using response surface methodology. Pharm. Dev. Technol 8:87-96 (2003).

20. G. L. Flynn. Influence of physico-chemical properties of drug and system on release of drugs from inert matrices. In A. C. Tanquary, and R. E. Lacey (eds.), Controlled release of biologically active agent, Plenum, New York, NY, 1974, pp. 73-98.

21. C. C. Wang. Mechanical properties and compaction properties of pharmaceutical pellets. Ph.D. thesis, University of Texas at Austin, Austin, TX, 1997.

22. H. Kuno and J. Okada. The compaction process and deformability of granules. Powder Technol 33:73-79 (1982). 